\title{
Identification and Autotuning of Temperature-Control System With Application to Injection Molding
}

\author{
Tao Liu, Ke Yao, and Furong Gao
}

\begin{abstract}
Motivated by the practical barrel-temperature control of injection molding, this paper proposes two identification methods and a united control scheme for general temperature control design. Based on the unity step response, corresponding to the full heating response in a temperature-control system, an identification method is developed to obtain an integrating model for heating-up control design. By using a relay test around the setpoint temperature, another identification method is proposed to obtain a model of stable or integrating type for control-system design to reject load disturbances during system operation. To ensure identification robustness, denoising strategies have also been presented for practical applications with measurement noise. For simplicity of implementation, a united control scheme based on the internal-model-control structure is proposed for both heating-up and steady operations against load disturbance. Analytical controller-design formulas and tuning rules are correspondingly developed for quantitative regulation of the heating-up response and the load-disturbance response for operation. Examples from the recent literature and a practical application to injection molding are performed to demonstrate the effectiveness and merit of the proposed identification methods and control scheme.
\end{abstract}

Index Terms-Injection-molding machine, integrating model, internal model control (IMC), relay test, step-response identification, temperature control.

\section{INTRODUCTION}

$\mathbf{T}$ EMPERATURE control is an important issue in many industrial processes, e.g., electric-resistance furnaces, crystal ovens, and heating boilers/tanks/barrels for various chemical and metallic products. Such a thermal process usually shows an integrating response characteristic during the heating stage, and after rising up to the set-point temperature, it tends to behave in a stable manner given a certain heating range, due to air convection or radiation loss into the environment. The main control challenges for such processes are to avoid overheating (i.e., temperature overshoot) in the heating stage and to tightly maintain the set-point temperature against load disturbances and process/environmental variations. Furthermore, thermal

Manuscript received December 18, 2007; revised August 23, 2008. Manuscript received in final form September 11, 2008. First published May 19, 2009; current version published October 23, 2009. This work was supported in part by the Hong Kong Research Grants Council under Project No. 613107 and in part by the Nansha Government under Project No. CPP-08-GD-001A0. Recommended by Associate Editor S.-L. Jämsä-Jounela.

T. Liu and F. Gao are with the Department of Chemical and Biomolecular Engineering, Hong Kong University of Science and Technology, Kowloon, Hong Kong (e-mail: liurouter@ieee.org; kefgao@ust.hk).

K. Yao is with the Center for Polymer Processing and Systems, Hong Kong University of Science and Technology, Kowloon, Hong Kong (e-mail: keyao@ust.hk).

Digital Object Identifier 10.1109/TCST.2008.2006746 processes typically have slow time constants and long time delay, causing difficulties to control-system design [1]. For instance, in the heating barrel of an injection-molding machine, as shown in Fig. 1, the raw materials (plastic resin) fed from the hopper need to be heated and melted to a temperature above 200 ${ }^{\circ} \mathrm{C}$ before injection molding. To ensure consistency in product quality, the front three zones (i.e., 1-3) of the heating barrel are required to be heated up with minimal temperature overshoot as quickly as possible and, then, maintain the melting temperature, e.g., $220^{\circ} \mathrm{C}$, for injection molding. Based on a priori knowledge of the barrel thermal inertia, recent literature [2] developed an optimal time switching strategy for heating-up with a small temperature overshoot. Several model-predictive-control methods [3]-[5] have been recently reported for effectively maintaining the barrel temperature. Moon [6], Kaymak and Luyben [7], and Wolff and Skogestad [8] developed multiloop control strategies for improving the load-disturbance-rejection performance. An iterative-learning-control scheme was proposed in [9] to guarantee temperature uniformity during system operation. Valigi et al.[10] and Shahrokhi and Nejati [11] discussed some implementation constraints in temperature control design. Grassi and Tsakalis [12] proposed a frequency loop-shaping method for controller tuning to accommodate for such implementational constraints. As illustrated in the recent papers [13]-[15], model-based control methods facilitate the improvement of system performance for heating-up and steady-state load-disturbance rejection. Most of the existing papers, e.g., [13]-[17], have been devoted to identification algorithms for obtaining stable first-order-plus-dead-time (FOPDT) or second-order-plus-dead-time (SOPDT) models, which, in fact, are confined to describe the process-response characteristics around the set-point temperature.

In this paper, a step-response identification method is proposed to obtain an integrating model for better depiction of the heating-up response characteristics, thus facilitating the heating-up control design. To reliably capture the process-response characteristics around the set-point temperature, a relay-based identification method is developed to obtain a stable or integrating SOPDT model for control-system design for steady-state load-disturbance rejection. For simplicity of implementation, a united control scheme for both heating-up and load-disturbance rejection is proposed based on the internal-model-control (IMC) structure. The corresponding controller-design formulas are analytically derived, and quantitative tuning rules are developed for obtaining the desired heating-up response and load-disturbance response. This paper is organized as follows. Section II presents the identification methods for obtaining the process models of heating-up and steady operations. A united control scheme for heating-up 


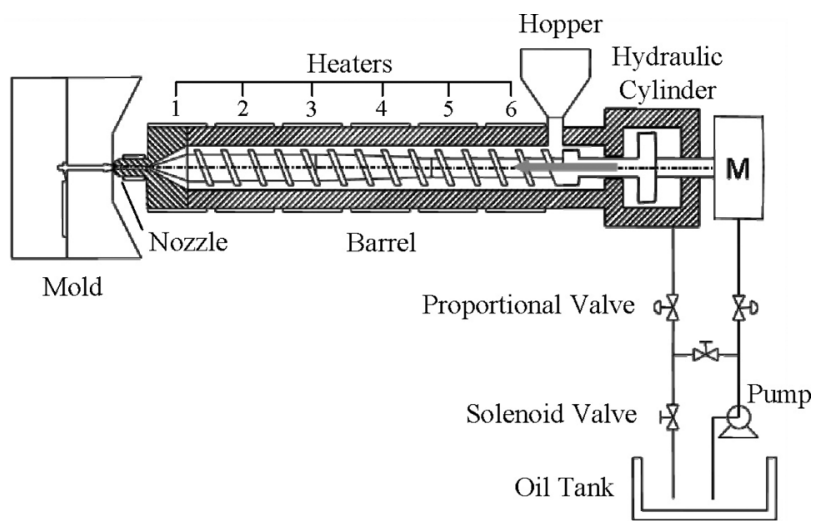

Fig. 1. Schematic diagram of an injection-molding machine.

and load-disturbance rejection is proposed in Section III, together with the corresponding controller-tuning guidelines. In Section IV, four examples in the recent literature are used to demonstrate effectiveness and merit of the proposed identification methods. Experimental tests on the barrel-temperature control of an injection-molding machine are performed in Section V. Finally, conclusions are drawn in Section VI.

\section{IDENTIFICATION METHODS}

To implement a quick heating-up to the set-point temperature, it is common to turn on the heater fully in the initial heating stage and, then, gradually decrease the heating power to reach the set-point temperature with as little as possible (or no) overheating. Accordingly, the integrating response under full heating is suitable for model identification for heating-up control design, which may be transformed to a unity step response for identification. An identification method based on the step response is therefore proposed. After the plant is heated up to the set-point temperature, full heating power is rarely needed, and different amounts of heating power may result in different response characteristics. In view of that, relay-feedback test is suitable for online identification [18], [19] and can be used to observe the repeatable response characteristics around the setpoint temperature; a relay-based identification method is proposed to capture the fundamental response characteristics at the steady state for control-system design. For clarity, the two identification methods are detailed, respectively, in the following two sections.

\section{A. Integrating Model for Heating-Up}

An integrating process can generally be modeled in the form of

$$
G_{\mathrm{m}-\mathrm{h}}=\frac{k_{\mathrm{p}} \mathrm{e}^{-\theta s}}{s\left(\tau_{\mathrm{p}} s+1\right)}
$$

where $k_{\mathrm{p}}$ is the process gain, $\theta$ is the time delay, and $\tau_{\mathrm{p}}$ is the time constant. Note that if $\tau_{\mathrm{p}} \rightarrow 0$, the above model may also represent a first-order integrating process.

For a step input, i.e., $u=\mu$ for $t>0$ and $u=0$ for $t \leq 0$, where $\mu \in \mathbb{R}$, the time-domain response of an inte- grating process described in (1) with zero initial condition can be derived as

$$
\begin{cases}y(t)=0, & 0<t \leq \theta \\ \tau_{\mathrm{p}} \ddot{y}(t)+\dot{y}(t)=k_{\mathrm{p}} \mu(t-\theta), & t>\theta .\end{cases}
$$

Triply integrating both sides of (2) for $t>\theta$ yields

$$
\int_{0}^{t} \int_{0}^{\tau_{2}} y\left(\tau_{1}\right) \mathrm{d} \tau_{1} \mathrm{~d} \tau_{2}=-\tau_{\mathrm{p}} \int_{0}^{t} y(\tau) \mathrm{d} \tau+\frac{1}{6} k_{\mathrm{p}} \mu(t-\theta)^{3}
$$

which can be rewritten as

$$
\psi(t)=\phi^{\mathrm{T}}(t) \nu
$$

where

$$
\begin{aligned}
& \psi(t)=\int_{0}^{t} \int_{0}^{\tau_{2}} y\left(\tau_{1}\right) \mathrm{d} \tau_{1} \mathrm{~d} \tau_{2} \\
& \phi(t)=\left[\mu t^{3} / 6, \quad-\mu t^{2} / 2, \quad \mu t / 2, \quad-\mu / 6, \quad-\int_{0}^{t} y(\tau) \mathrm{d} \tau\right]^{\mathrm{T}}
\end{aligned}
$$

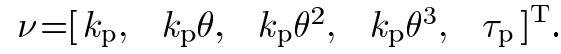

In view of that $\psi(t)=y(t)=0$ for $t \leq \theta$, we shall choose the time sequence $t_{i}(i=1,2, \ldots, N)$, with a limitation of $\theta \leq t_{1}<t_{2}<\cdots<t_{N}$ for model fitting. In fact, the process time delay may not be explicitly known beforehand, particularly in the presence of measurement noise. It is thus suggested to choose $t_{1}$ slightly larger than the observed response delay from the test. Then, by letting $\Psi=\left[\psi\left(t_{1}\right), \psi\left(t_{2}\right), \ldots, \psi\left(t_{N}\right)\right]^{\mathrm{T}}$ and $\Phi=\left[\phi\left(t_{1}\right), \phi\left(t_{2}\right), \ldots, \phi\left(t_{N}\right)\right]^{\mathrm{T}}$, a least squares (LS) algorithm for parameter estimation can be established as

$$
\nu=\left(\Phi^{\mathrm{T}} \Phi\right)^{-1} \Phi \Psi
$$

It can be easily verified that each column of $\Phi$ is linearly independent with each other, such that $\Phi$ is guaranteed nonsingular for computation of (5). Accordingly, there exists a unique solution of $\nu$ for the time sequence. For practical applications, the number of data points $(N)$ for fitting should be in the range of 50-200 for a good tradeoff between computation efficiency and fitting accuracy.

Then, the process time constant can be retrieved as

$$
\tau_{\mathrm{p}}=\nu(5)
$$

Note that, besides $k_{\mathrm{p}}=v(1)$ and $k_{\mathrm{p}} \theta=v(2)$, there exist two redundant fitting conditions, $k_{\mathrm{p}} \theta^{2}=v(3)$ and $k_{\mathrm{p}} \theta^{3}=v(4)$, for determining the process gain and time delay. To procure identification accuracy for a real high-order process, we may take the natural logarithm for both sides of the four conditions to obtain

$$
\left[\begin{array}{ll}
1 & 0 \\
1 & 1 \\
1 & 2 \\
1 & 3
\end{array}\right]\left[\begin{array}{c}
\ln k_{p} \\
\ln \theta
\end{array}\right]=\left[\begin{array}{l}
\ln \nu(1) \\
\ln \nu(2) \\
\ln \nu(3) \\
\ln \nu(4)
\end{array}\right] .
$$

Thus, $k_{\mathrm{p}}$ and $\theta$ can be retrieved from (7) using an LS fitting algorithm.

Remark 1: By doubly integrating both sides of (2) for $t>$ $\theta$, an identification algorithm with less computation effort can be similarly obtained, but the identification robustness against measurement noise may be inferior to the above algorithm that 
utilizes the integral function to reduce the measurement errors according to the statistical averaging principle. Note that, by letting $\tau_{\mathrm{p}}=0$, the above algorithm can be transparently generalized to identify an FOPDT integrating-process model, $G_{\mathrm{m}-\mathrm{h}}=$ $k_{\mathrm{p}} \mathrm{e}^{-\theta s} / s$, which, however, is inferior to an SOPDT model for representing a real high-order process, since the initial transient response cannot be effectively represented. A detailed demonstration can be found in a recent paper [20]. Besides, it should be noted that the step response of an integrating process will increase infinitely as $t \rightarrow \infty$ and, thus, should be limited within an admissible range around the set-point value. Obviously, a larger range of the step response, corresponding to a longer time sequence, facilitates better identification accuracy.

In the case that the process-output measurement is corrupted by random noise $\zeta(t)$ during the step-response test, there exists $\hat{y}(t)=y(t)+\zeta(t)$, where $\hat{y}(t)$ denotes the corrupted output. It follows from (3) that

$$
\Psi=\Phi \nu+\sigma
$$

where $\sigma=\left[\delta\left(t_{1}\right), \delta\left(t_{2}\right), \ldots, \delta\left(t_{N}\right)\right]^{\mathrm{T}}$ and $\delta(t)=$ $\int_{0}^{t} \int_{0}^{\tau_{2}} \zeta\left(\tau_{1}\right) \mathrm{d} \tau_{1} \mathrm{~d} \tau_{2}+\tau_{\mathrm{p}} \int_{0}^{t} \zeta(\tau) \mathrm{d} \tau$. Due to the fact that $\Phi$ is now correlated with $\sigma$, the LS estimate given in (5) may not be consistent according to the parameter-estimation theory [21]. One solution is to use the instrumental-variable (IV) method [21], [22]. A feasible choice of such an IV matrix is given in the following proposition

Proposition 1: If the IV matrix is chosen as $Z=$ $\left[z_{1}, z_{2}, \ldots, z_{N}\right]^{\mathrm{T}}$ with $z_{i}=\left[1 / t_{i}^{2}, 1 / t_{i}, 1, t_{i}, t_{i}^{2}\right]^{\mathrm{T}}$ for $i=1,2, \ldots, N$, which satisfies the two limiting conditions: 1) The inverse of $\lim _{N \rightarrow \infty}\left(Z^{\mathrm{T}} \Phi\right) / N$ exists and 2) $\lim _{N \rightarrow \infty}\left(Z^{\mathrm{T}} \sigma\right) / N=0$, then a consistent estimation for (8) is $\nu=\left(Z^{\mathrm{T}} \Phi\right)^{-1} Z \Psi$.
Proof: The limiting conditions in Proposition 1 are sufficient for consistent estimation [21]. We therefore must demonstrate that the proposed IV matrix satisfies them. For the first condition, we can derive (9), shown at the bottom of the page. Note that

$$
\lim _{N \rightarrow \infty} \frac{t_{N}}{N}=c
$$

where $c \in \mathbb{R}_{+}$. For instance, $c=T_{\mathrm{S}}$ is for the case that $t_{i}=$ $t_{1}+T_{\mathrm{s}}(i-1)(i=1,2, \ldots, N)$, where $T_{\mathrm{s}}$ is the sampling period for identification. Therefore, all rows or columns in the square matrix of (9) are nonzero vectors and linearly independent of each other, which corresponds to

$$
\operatorname{det}\left(\lim _{N \rightarrow \infty} \frac{Z^{\mathrm{T}} \Phi}{N}\right) \neq 0
$$

Hence, the first condition in Proposition 1 is satisfied.

For the second condition, we can derived (12), shown at the bottom of the page. Note that, for $N \rightarrow \infty$, the measurement noise sequence $\zeta\left(t_{i}\right)(i=1,2, \ldots, N)$ can be viewed as white noise such that its mean value tends to zero. Accordingly, it follows from $\delta(t)=\int_{0}^{t} \int_{0}^{\tau_{2}} \zeta\left(\tau_{1}\right) \mathrm{d} \tau_{1} \mathrm{~d} \tau_{2}+\tau_{\mathrm{p}} \int_{0}^{t} \zeta(\tau) \mathrm{d} \tau$ that

$$
\lim _{N \rightarrow \infty} \sum_{i=1}^{N} \delta\left(t_{i}\right)=0
$$

which indicates that $\delta\left(t_{i}\right)(i=1,2, \ldots, N)$ is also a random noise sequence for $N \rightarrow \infty$ and, thus, is uncorrelated with the time sequence $t_{i}$ or $t_{i}^{2}(i=1,2, \ldots, N)$. Hence, the last two elements in the vector of (12) also become zero for $N \rightarrow \infty$. This completes the proof.

$$
\lim _{N \rightarrow \infty} \frac{Z^{\mathrm{T}} \Phi}{N}=\left[\begin{array}{cccc}
\frac{\mu}{6} \lim _{N \rightarrow \infty} \frac{1}{N} \sum_{i=1}^{N} t_{i} & -\frac{\mu}{2} & 0 & 0 \\
\frac{\mu}{6} \lim _{N \rightarrow \infty} \frac{1}{N} \sum_{i=1}^{N} t_{i}^{2} & -\frac{\mu}{2} \lim _{N \rightarrow \infty} \frac{1}{N} \sum_{i=1}^{N} t_{i} & \frac{\mu}{2} & 0 \\
\frac{\mu}{6} \lim _{N \rightarrow \infty} \frac{1}{N} \sum_{i=1}^{N} t_{i}^{3} & -\frac{\mu}{2} \lim _{N \rightarrow \infty} \frac{1}{N} \sum_{i=1}^{N} t_{i}^{2} & \frac{\mu}{2} \lim _{N \rightarrow \infty} \frac{1}{N} \sum_{i=1}^{N} t_{i} & -\frac{\mu}{6} \\
\frac{\mu}{6} \lim _{N \rightarrow \infty} \frac{1}{N} \sum_{i=1}^{N} t_{i}^{4} & -\frac{\mu}{2} \lim _{N \rightarrow \infty} \frac{1}{N} \sum_{i=1}^{N} t_{i}^{3} & \frac{\mu}{2} \lim _{N \rightarrow \infty} \frac{1}{N} \sum_{i=1}^{N} t_{i}^{2} & -\frac{\mu}{6} \lim _{N \rightarrow \infty} \frac{1}{N} \sum_{i=1}^{N} t_{i}-\lim _{N \rightarrow \infty} \frac{1}{N} \int_{0}^{t_{i}} y(\tau) \mathrm{d} \tau \\
\frac{\mu}{6} \lim _{N \rightarrow \infty} \frac{1}{N} \sum_{i=1}^{N} t_{i}^{5} & -\frac{\mu}{2} \lim _{N \rightarrow \infty} \frac{1}{N} \sum_{i=1}^{t_{i}} t_{i}^{4} & \frac{\mu}{2} \lim _{N \rightarrow \infty} \frac{1}{N} \sum_{i=1}^{N} t_{i}^{3} & -\frac{\mu}{6} \lim _{N \rightarrow \infty} \frac{1}{N} \sum_{i=1}^{2} t_{i}^{2}-\lim _{N \rightarrow \infty} \frac{t_{i}^{2}}{N} \int_{0}^{t_{i}} y(\tau) \mathrm{d} \tau
\end{array}\right] .
$$

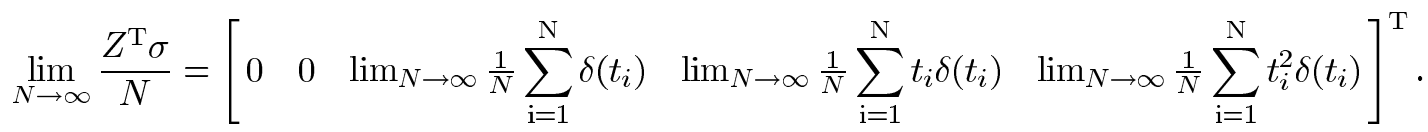




\section{B. Stable/Integrating Model for Set-Point Operation}

To describe the approximately stable response characteristics around the set-point temperature, the following stable SOPDT process model is used

$$
G_{\mathrm{m}-\mathrm{s}}(s)=\frac{k_{\mathrm{p}} \mathrm{e}^{-\theta s}}{\left(\tau_{\mathrm{p}} s+1\right)^{2}} .
$$

This model is customarily called a critically damped type. It should be noted that the other two SOPDT stable model types, underdamped and overdamped, are no more suitable for describing such temperature response besides with one more parameter. They are thus not adopted here.

In a relay-feedback test for identification, the relay function is generally specified as

$$
\begin{aligned}
& u(t) \\
& = \begin{cases}u_{+}, & \text {for }\left\{e(t)>\varepsilon_{+}\right\} \text {or }\left\{e(t) \geq \varepsilon_{-} \text {and } u\left(t_{-}\right)=u_{+}\right\} \\
u_{-}, & \text {for }\left\{e(t)<\varepsilon_{-}\right\} \text {or }\left\{e(t) \leq \varepsilon_{+} \text {and } u\left(t_{-}\right)=u_{-}\right\}\end{cases}
\end{aligned}
$$

where $u_{+}$and $u_{-}$are, respectively, the positive and negative relay-output magnitudes and $\varepsilon_{+}$and $\varepsilon_{-}$are, respectively, the positive and negative relay-switch hystereses. By letting $u_{+}=$ $\left|u_{-}\right|$and $\varepsilon_{+}=\left|\varepsilon_{-}\right|$, an unbiased (symmetrical) relay can be obtained. For identification of the temperature response, $u_{+}$corresponds to the heating power that may be specified to increase the plant temperature within an admissible range, and $u_{-}$may simply be specified as zero that corresponds to shutting off the heater for cooling via air convection or radiation; $\varepsilon_{+}$and $\varepsilon_{-}$ correspond to user-specified temperatures for the relay switch between heating and cooling around the set-point temperature. To avoid measurement noise causing incorrect relay switches, a short "listening period" (e.g., 20-100 samples) may be adopted for reference to set $\varepsilon_{+}$and $\varepsilon_{-}$properly in practice.

In a relay test, the temperature response moves into a limit cycle, i.e., the temperature response becomes a periodic function with respect to the angular frequency of the oscillation, $\omega_{\mathrm{u}}=2 \pi / P_{\mathrm{u}}$, where $P_{\mathrm{u}}$ is the oscillation period. Using the idea of time shift, we may view the temperature response as a periodic response from the very beginning, so its Fourier transform can be derived as

$$
\begin{aligned}
Y\left(j \omega_{\mathrm{u}}\right) & =\lim _{n \rightarrow \infty} n \int_{0}^{P_{\mathrm{u}}} y_{\mathrm{os}}(t) \mathrm{e}^{-j \omega_{\mathrm{u}} t} \mathrm{~d} t \\
& =\lim _{n \rightarrow \infty} n \int_{t_{\mathrm{os}}}^{t_{\mathrm{os}}+P_{\mathrm{u}}} y(t) \mathrm{e}^{-j \omega_{\mathrm{u}} t} \mathrm{~d} t
\end{aligned}
$$

where $y_{\mathrm{os}}(t)$ denotes the output temperature for $t \in\left[t_{\mathrm{os}}, \infty\right)$, and $t_{\mathrm{os}}$ may be taken as any relay-switch point in the steady oscillation, such that the influence from the initial temperature response to the above periodic integral can be excluded.

Similarly, the Fourier transform of the relay output can be expressed as

$$
U\left(j \omega_{\mathrm{u}}\right)=\lim _{n \rightarrow \infty} n \int_{t_{\mathrm{os}}}^{t_{\mathrm{os}}+P_{\mathrm{u}}} u(t) \mathrm{e}^{-j \omega_{\mathrm{u}} t} \mathrm{~d} t .
$$

Thereby, the temperature response with respect to $\omega_{\mathrm{u}}$ can be derived as

$$
G\left(j \omega_{\mathrm{u}}\right)=\frac{Y\left(j \omega_{\mathrm{u}}\right)}{U\left(j \omega_{\mathrm{u}}\right)}=\frac{\int_{t_{\mathrm{os}}}^{t_{\mathrm{os}}+P_{\mathrm{u}}} y(t) \mathrm{e}^{-j \omega_{\mathrm{u}} t \mathrm{~d} t}}{\int_{t_{\mathrm{os}}}^{t_{\mathrm{os}}+P_{\mathrm{u}}} u(t) \mathrm{e}^{-j \omega_{\mathrm{u}} t} \mathrm{~d} t}=A_{\mathrm{u}} \mathrm{e}^{j \varphi_{\mathrm{u}}} .
$$

In view of that, the relay test for identification of the temperature response is obviously biased; the model gain can be separately derived similar to (18) from

$$
k_{\mathrm{p}}=G(0)=\frac{\int_{t_{\mathrm{os}}}^{t_{\mathrm{os}}+P_{\mathrm{u}}} y(t) \mathrm{d} t}{\int_{t_{\mathrm{os}}}^{t_{\mathrm{os}}+P_{\mathrm{u}}} u(t) \mathrm{d} t} .
$$

Substituting the process model of (14) into (18) yields the frequency-response fitting conditions

$$
\begin{aligned}
& A_{\mathrm{u}}=\frac{k_{\mathrm{p}}}{\tau_{\mathrm{p}}^{2} \omega_{\mathrm{u}}^{2}+1} \\
& -\theta \omega_{\mathrm{u}}-2 \arctan \left(\tau_{\mathrm{p}} \omega_{\mathrm{u}}\right)=\varphi_{\mathrm{u}} .
\end{aligned}
$$

Hence, $\tau_{\mathrm{p}}$ and $\theta$ can be, respectively, derived from (20) and (21) as

$$
\begin{aligned}
\tau_{\mathrm{p}} & =\frac{1}{\omega_{\mathrm{u}}} \sqrt{\frac{k_{\mathrm{p}}}{A_{\mathrm{u}}}-1} \\
\theta & =-\frac{1}{\omega_{\mathrm{u}}}\left[\varphi_{\mathrm{u}}+2 \arctan \left(\tau_{\mathrm{p}} \omega_{\mathrm{u}}\right)\right] .
\end{aligned}
$$

In the case that a relatively larger heating power causes the temperature response to behave in an integrating manner around the set-point temperature, an integrating model of (1) may be necessary to design the control system for steady-state load-disturbance rejection. In such a case, the model gain cannot be derived from (19) because $G(0) \rightarrow \infty$. However, the time delay of the temperature response can be intuitively measured from the initial step response for heating or cooling in a relay test. For instance, after the process maintains the set-point temperature with a certain heating power, the time from shutting off the heater for the relay test to the beginning of the temperature drop can be counted as the time delay of the temperature response. Consequently, by substituting the integrating model of (1) into the frequency-response fitting condition of (18), $k_{\mathrm{p}}$ and $\tau_{\mathrm{p}}$ can then be derived as

$$
\begin{aligned}
& k_{\mathrm{p}}=A_{\mathrm{u}} \omega_{\mathrm{u}} \sqrt{\tau_{\mathrm{p}}^{2} \omega_{\mathrm{u}}^{2}+1} \\
& \tau_{\mathrm{p}}=\frac{1}{\omega_{\mathrm{u}}} \tan \left(-\theta \omega_{\mathrm{u}}-\frac{\pi}{2}-\varphi_{\mathrm{u}}\right) .
\end{aligned}
$$

It should be noted that, by using the time delay measured from the initial relay response, $k_{\mathrm{p}}$ and $\tau_{\mathrm{p}}$ of the stable model shown in (14) can alternatively be derived from (20) and (21) as

$$
\begin{aligned}
k_{\mathrm{p}} & =A_{\mathrm{u}}\left(\tau_{\mathrm{p}}^{2} \omega_{\mathrm{u}}^{2}+1\right) \\
\tau_{\mathrm{p}} & =\frac{1}{\omega_{\mathrm{u}}} \tan \left(-\frac{\theta \omega_{\mathrm{u}}+\varphi_{\mathrm{u}}}{2}\right) .
\end{aligned}
$$

To cope with measurement noise occurring in such a relay test, the frequency-response estimation of (18) may be computed from the averaging of 5-20 repetitive or similar oscillation periods. When the measurement noise causes a high noise-tosignal ratio (NSR) level, a low-pass Butterworth filter may be 


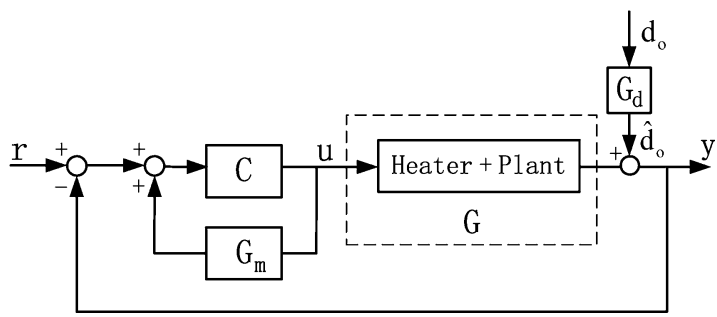

Fig. 2. Block diagram of the proposed temperature-control structure.

used for the output measurement and relay feedback. In view of the fact that the measurement noise is mainly of high frequency, the guideline for choosing the cutoff frequency is given as

$$
f_{\mathrm{c}} \geq 5 f_{\mathrm{u}}=\frac{5}{P_{\mathrm{u}}}
$$

In the result, only the signal components within the frequency band around $f_{\mathrm{u}}$ can be passed through. Note that the phase lag caused by the low-pass filter almost does not affect the measurement of the oscillation amplitude and the period of the limit cycle, because the relay output has a similar phase lag under the filtered feedback. In fact, further improved denoising effect, e.g., no phase lag and magnitude deviation, can be obtained by using an offline denoising strategy, i.e., filtering the resulting limit-cycle data of the relay test in both the forward and reverse directions with the same low-pass filter.

\section{CONTROL-SYSTEM DESIGN}

For simplicity of implementation, a united control scheme based on the IMC structure is proposed for both heating-up and steady-state operations, which is shown in Fig. 2, where the plant plus the heater are treated as the process (denoted as $G)$ to be controlled, $G_{\mathrm{m}}$ is the process model thereby identified, $C$ is the controller, $r$ is the set-point temperature, $y$ is the measured plant temperature, and $d_{0}$ is the load disturbance with a transfer function of $G_{\mathrm{d}}$, including air convection and heat absorption from the raw materials. The corresponding controller-tuning methods are given in the following two sections.

\section{A. Heating-Up Control}

From Fig. 2, the closed-loop transfer function can be derived as

$$
T=\frac{G C}{1+\left(G-G_{\mathrm{m}}\right) C} .
$$

In the nominal case, $G=G_{\mathrm{m}}$; the transfer function can be simplified to $T=G C$.

Based on the identified integrating SOPDT model of (1), the desired closed-loop transfer function is proposed in the form of

$$
T_{\mathrm{r}-\mathrm{h}}=\frac{\mathrm{e}^{-\theta s}}{\left(\lambda_{\mathrm{h}} s+1\right)^{2}}
$$

where $\lambda_{\mathrm{h}}$ is an adjustable closed-loop time constant. Note that the above closed-loop transfer function can contribute in achieving the $\mathrm{H}_{2}$ optimal performance for set-point tracking [23].
The corresponding closed-loop controller $C_{\mathrm{h}}$ can then be derived from the nominal relationship, $T_{\mathrm{r}-\mathrm{h}}=G_{\mathrm{m}-\mathrm{h}} C_{\mathrm{h}}$, i.e.,

$$
C_{\mathrm{h}}=\frac{s\left(\tau_{\mathrm{p}} s+1\right)}{k_{\mathrm{p}}\left(\lambda_{\mathrm{h}} s+1\right)^{2}} \text {. }
$$

It is seen that $\lambda_{\mathrm{h}}$ becomes the adjustable parameter of $C_{\mathrm{h}}$, which may be tuned to achieve a desirable heating-up specification.

For a step change of the set-point input, $\Delta r=r-y_{0}$, which corresponds to heating-up from the initial process temperature of $y_{0}$ to the desired temperature of $r$ (i.e., the set-point value), the time-domain response can be derived accordingly as

$$
y_{\mathrm{r}-\mathrm{h}}(t)= \begin{cases}0, & t \leq \theta \\ \Delta r\left[1-\left(1+\frac{t-\theta}{\lambda_{\mathrm{h}}}\right) \mathrm{e}^{-(t-\theta) / \lambda_{\mathrm{h}}}\right], & t>\theta\end{cases}
$$

which indicates that there is no temperature overshoot for heating-up in the nominal case (i.e., $G=G_{\mathrm{m}-\mathrm{h}}$ ), and quantitative time-domain performance specification can be conveniently determined by tuning the single adjustable parameter $\lambda_{\mathrm{h}}$ of $C_{\mathrm{h}}$. For instance, define the heating-up time $t_{\mathrm{h}}$ as the time to reach $95 \%$ of $\Delta r$; the tuning formula can be derived from (32) as $t_{\mathrm{h}}=4.7439 \lambda_{\mathrm{h}}+\theta$.

Note that, due to heat loss from air convection or radiation to the environment, which may be viewed as load disturbance, the control output should actually be augmented with a certain value to prevent the temperature drop during/after the heating-up stage, i.e.,

$$
u=u_{\mathrm{r}-\mathrm{h}}+u_{0}
$$

where $u_{0}$ corresponds to the heating power required for balancing the heat loss from air convection or radiation, which may be ascertained from an open-loop test of maintaining the set-point temperature.

In addition, there exists an implementation constraint of $0 \leq u \leq 1$, corresponding to $0-100 \%$ of the heater capacity. Checking the range of control output in tuning $\lambda_{\mathrm{h}}$ of $C_{\mathrm{h}}$ is thus necessary. In the case that the control output surpasses the upper bound for some moments in the heating-up response, the heating-up time will be prolonged, but the stability of the control system can still be preserved according to the IMC theory [24]. If the computed control output is smaller than the lower bound for some moments during the heating-up response, steady-state deviation of the output temperature may be yielded, as can be verified from simulation or experimental tests.

The load-disturbance transfer function from $d_{\mathrm{o}}$ to $y$ shown in Fig. 2 can be derived from

$$
H_{\mathrm{d}_{0}}(s)=G_{\mathrm{d}}(s)\left(1-T_{\mathrm{r}-\mathrm{h}}(s)\right)
$$

where $G_{\mathrm{d}}(s)$ is a stable transfer function that reflects the dynamics of load disturbance. It can be verified using (30) that

$$
\lim _{s \rightarrow 0} H_{\mathrm{d}}(s)=0
$$

which indicates that the influence of step-type load disturbance from the process-output side can be eliminated asymptotically. It should be noted that no step-type load disturbance could seep 


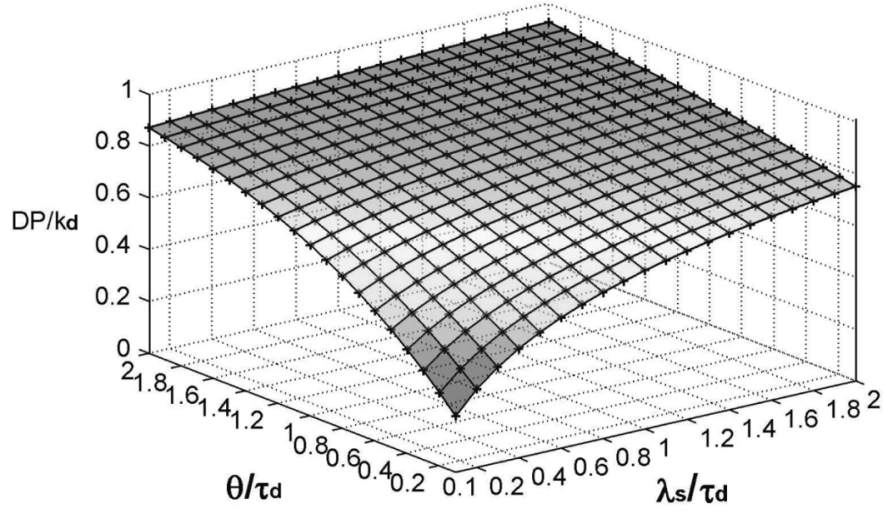

Fig. 3. Quantitative tuning relationship between the control parameter and DP.

into the process input which is purely manipulated by the controller as shown in Fig. 2, so the influence of such load disturbance does not need to be considered, although it may cause the control system unstability as discussed in [23].

To evaluate robust stability of the resulting control system, we may lump multiple sources of the process uncertainties into the form of multiplicative uncertainty, i.e., $\Delta_{\mathrm{m}}(s)=[G(s)-$ $\left.G_{\mathrm{m}}(s)\right] / G_{\mathrm{m}}(s)$, without loss of generality [25]. It follows from the small gain theorem that the control system holds robust stability if and only if

$$
\left\|\Delta_{\mathrm{m}}(s) T_{\mathrm{r}-\mathrm{h}}(s)\right\|_{\infty}<1 .
$$

Substituting (30) into (36) yields

$$
\sqrt{\left(\lambda_{\mathrm{h}}^{2} \omega^{2}+1\right)}>\left|\Delta_{m}(j \omega)\right| \quad \forall \omega \geq 0
$$

which can be verified by observing whether the magnitude plot of the left-hand side of (37) is larger than the right-hand side for $\omega \in[0, \infty)$. Therefore, given an upper bound of $\left|\Delta_{m}\right|$ in practice, e.g., the maximal variation of the model parameters, the admissible tuning range of $\lambda_{\mathrm{h}}$ can be determined from (37).

\section{B. Autotuning for Load-Disturbance Rejection}

After heating up to the set-point temperature, the controller tuning then aims to reject load disturbance. Based on the stable SOPDT model of (14) identified around the set-point temperature, the desired closed-loop transfer function is proposed to be similar to (30) as

$$
T_{\mathrm{r}-\mathrm{s}}=\frac{\mathrm{e}^{-\theta s}}{\left(\lambda_{\mathrm{s}} s+1\right)^{2}}
$$

where $\lambda_{\mathrm{s}}$ is an adjustable closed-loop time constant. Note that the above closed-loop transfer function can contribute to minimizing the integral-squared-error criterion for rejecting a step load disturbance that seeps into the process output (denoted as $\hat{d}_{\mathrm{o}}$ in Fig. 2) [23].

The corresponding controller $C_{\mathrm{s}}$ can then be derived from the nominal closed-loop relationship, $T_{\mathrm{r}-\mathrm{s}}=G_{\mathrm{m}-\mathrm{s}} C_{\mathrm{s}}$, i.e.,

$$
C_{\mathrm{s}}=\frac{\left(\tau_{\mathrm{p}} s+1\right)^{2}}{k_{\mathrm{p}}\left(\lambda_{\mathrm{s}} s+1\right)^{2}} \text {. }
$$

It is seen that $\lambda_{\mathrm{s}}$ becomes the adjustable parameter of $C_{\mathrm{s}}$, which may be tuned to obtain desirable disturbance-rejection performance.

To evaluate the achievable performance for load-disturbance rejection, an FOPDT transfer function of $G_{\mathrm{d}}(s)=$ $k_{\mathrm{d}} \mathrm{e}^{-\theta_{\mathrm{d}} s} /\left(\tau_{\mathrm{d}} s+1\right)$ may be used to represent approximately the dynamics of a load disturbance shown as $d_{\mathrm{o}}$ in Fig. 2, where $k_{\mathrm{d}}$ indicates the magnitude of $d_{\mathrm{o}}, \theta_{\mathrm{d}}$ is the time delay to affect the process output, and $\tau_{\mathrm{d}}$ is the time constant that reflects the changing speed of $d_{\mathrm{o}}$ if it has continuous power with respect to the time. Note that $\tau_{\mathrm{d}}=0$ corresponds to step change in the load disturbance. For a unity step change of $d_{\mathrm{o}}$, the time-domain disturbance response can be derived accordingly from(40), shown at the bottom of the page. It can be seen that if $\tau_{\mathrm{d}}=0$, then the disturbance-response peak (DP) is equal to $k_{\mathrm{d}}$. For $\tau_{\mathrm{d}}>0$, in view of that no analytical solution of DP can be derived from (40), numerical results are cultivated to disclose the quantitative relationship between DP and $\lambda_{\mathrm{s}}$. By using a frequency scaling factor, $\widehat{s}=\tau_{\mathrm{d}} s$, the unity step disturbance response can be normalized as

$$
y_{\mathrm{d}_{0}}\left(\frac{t}{\tau_{\mathrm{d}}}\right)=L^{-1}\left\{\tau_{\mathrm{d}} \cdot \frac{k_{\mathrm{d}} \mathrm{e}^{-\widehat{\theta}_{\mathrm{d}} \widehat{s}}}{\widehat{s}(\widehat{s}+1)} \times\left[1-\frac{\mathrm{e}^{-\widehat{\theta} \widehat{s}}}{\left(\widehat{\left.\lambda_{\mathrm{s}} \widehat{s}+1\right)^{2}}\right.}\right]\right\}_{(41)}
$$

where $\widehat{\theta}_{\mathrm{d}}=\theta_{\mathrm{d}} / \tau_{\mathrm{d}}, \widehat{\theta}=\theta / \tau_{\mathrm{d}}$, and $\widehat{\lambda}_{\mathrm{s}}=\lambda_{\mathrm{s}} / \tau_{\mathrm{d}}$. It is therefore seen that $y_{\mathrm{d}_{0}}(t)$ has the same DP with $y_{\mathrm{d}_{0}}\left(t / \tau_{\mathrm{d}}\right)$. Note that $\theta_{\mathrm{d}}$ (or $\widehat{\theta}_{\mathrm{d}}$ ) does not affect DP. Based on numerical simulations for $y_{\mathrm{d}_{0}}\left(t / \tau_{\mathrm{d}}\right)$, the quantitative tuning relationship between $\mathrm{DP} / k_{\mathrm{d}}, \lambda_{\mathrm{s}} / \tau_{\mathrm{d}}$, and $\theta / \tau_{\mathrm{d}}$ is shown in Fig. 3. In practice, it is suggested to let $\lambda_{\mathrm{s}}=\theta$ initially, and then, by monotonically increasing or decreasing $\lambda_{\mathrm{s}}$ online, a desirable performance specification for load-disturbance rejection can be conveniently determined.

Following the robust stability analysis given in the earlier section, the robust tuning constraint for holding the control-system stability can be similarly derived from

$$
\sqrt{\left(\lambda_{\mathrm{s}}^{2} \omega^{2}+1\right)}>\left|\Delta_{m}(j \omega)\right| \quad \forall \omega \geq 0
$$

$$
y_{\mathrm{d}_{0}}(t)= \begin{cases}0, & t \leq \theta_{\mathrm{d}} \\ k_{\mathrm{d}}\left(1-\mathrm{e}^{-\frac{t-\theta_{\mathrm{d}}}{\tau_{\mathrm{d}}}}\right), & \theta_{\mathrm{d}}<t \leq \theta_{\mathrm{d}}+\theta \\ k_{\mathrm{d}}\left[\mathrm{e}^{-\frac{t-\theta_{\mathrm{d}}-\theta}{\lambda_{\mathrm{s}}}}-\mathrm{e}^{-\frac{t-\theta_{\mathrm{d}}}{\tau_{\mathrm{d}}}}+\frac{\tau_{\mathrm{d}}^{2}}{\left(\lambda_{\mathrm{s}}-\tau_{\mathrm{d}}\right)^{2}}\left(\mathrm{e}^{-\frac{t-\theta_{\mathrm{d}}-\theta}{\tau_{\mathrm{d}}}}-\mathrm{e}^{-\frac{t-\theta_{\mathrm{d}}-\theta}{\lambda_{\mathrm{s}}}}\right)-\frac{t-\theta_{\mathrm{d}}-\theta}{\lambda_{\mathrm{s}}-\tau_{\mathrm{d}}} \mathrm{e}^{-\frac{t-\theta_{\mathrm{d}}-\theta}{\lambda_{\mathrm{s}}}}\right], & t>\theta_{\mathrm{d}}+\theta\end{cases}
$$


TABLE I

RELAY IDENTIFICATION FOR EXAMPLES 2 AND 4

\begin{tabular}{ccccc}
\hline & \multicolumn{3}{c}{ Limit Cycle } & \\
\cline { 2 - 4 } Process & $P_{\mathrm{u}}$ & $A_{\mathrm{u}}$ & $\varphi_{\mathrm{u}}$ & \\
\hline$\frac{e^{-s}}{(s+1)^{2}}$ & 5.89 & 0.4677 & -2.7075 & $\frac{1.0000 e^{-1.0049 s}}{(1.0000 s+1)^{2}}$ \\
$\frac{e^{-0.5 s}}{(s+1)\left(s^{2}+s+1\right)}$ & 6.24 & 0.6998 & -2.8819 & $\frac{1.0000 e^{-1.7103 s}}{(0.6505 s+1)^{2}}$ \\
\hline
\end{tabular}

which may be used to ascertain the admissible tuning range of $\lambda_{\mathrm{s}}$ in the presence of the process uncertainties.

For the case that the integrating model of (1) is identified from a relay test or the aforementioned step response, the controller-design method for heating-up can also be used for loaddisturbance rejection, by virtue of the united control structure. Note that the closed-loop sensitivity function $H$ satisfies the following asymptotic constraint for rejecting a step load disturbance that seeps into the process output

$$
\lim _{s \rightarrow 0} H(s)=\lim _{s \rightarrow 0}(1-T(s))=0
$$

which can be verified by substituting (30) or (38) into (43). Even for the case that the controller is designed based on the stable model of (14), whereas the temperature response actually behaves in an integrating manner, e.g., described by the integrating model of (1), the asymptotic constraint of (43) can still be satisfied, which can be verified by substituting (14), (29), and (39) into (43). In such a case, the model mismatch can be assessed with the aforementioned multiplicative uncertainty, and correspondingly, the tuning constraint for holding system stability can be determined in terms of the earlier robust stability analysis.

\section{Simulation EXAMPLES}

Four examples from the recent literature are used to illustrate the effectiveness of the proposed identification methods: Examples 1 and 2 are given to demonstrate that high accuracy can be obtained by the proposed methods for identification of secondorder integrating and stable processes, together with measurement noise tests for demonstrating identification robustness, and Examples 3 and 4 are given to show the achievable fitting for high-order integrating and stable processes in comparison with results from the recent literature.

Example 1: Consider the SOPDT integrating process studied in [26]

$$
G=\frac{\mathrm{e}^{-10 s}}{s(20 s+1)}
$$

Based on the measured output data in the time interval of $[8,30]$ (in seconds) under a unity step-response test, the proposed identification method composed of (4), (5), (6), and (7) gives the following process model, $G_{\mathrm{m}}=1.0000 \mathrm{e}^{-10.01 s} / s(20.0000 s+1)$, indicating good accuracy.

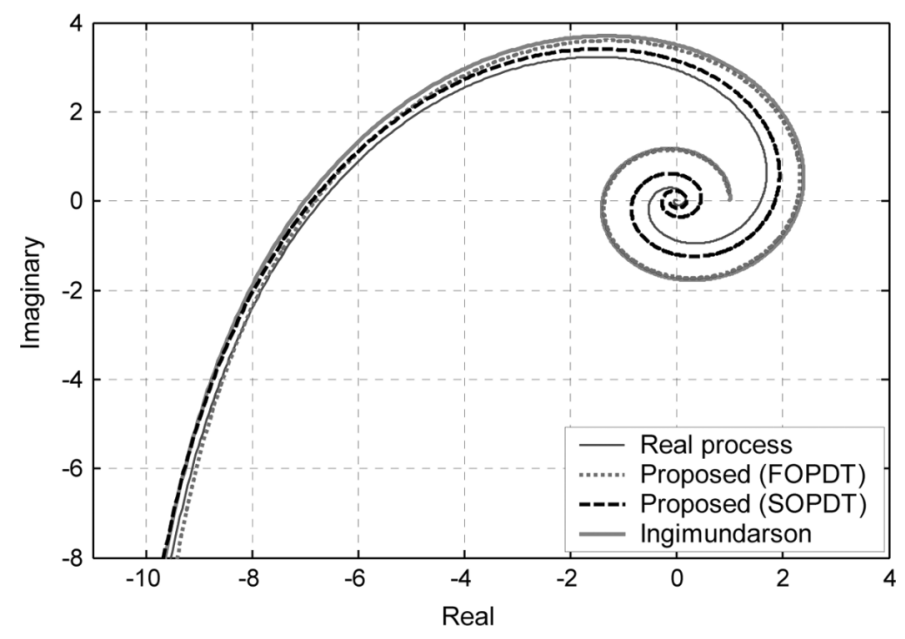

Fig. 4. Nyquist plots of model fitting for Example 3.

Now, suppose that random noise of $N\left(0, \sigma_{N}^{2}=0.0127\right)$ is added to the process-output measurement, causing NSR $=5 \%$. Using the measured output data in the aforementioned time interval, the proposed IV identification methods result in the process model, $G_{\mathrm{m}}=1.0362 \mathrm{e}^{-9.8205 s} / s(21.1995 s+1)$. According to the widely used output error criterion, err $=\sum_{k=1}^{N}\left[y\left(k T_{\mathrm{s}}\right)-\widehat{y}\left(k T_{\mathrm{s}}\right)\right]^{2} / N$, where $y\left(k T_{\mathrm{s}}\right)$ and $\widehat{y}\left(k T_{\mathrm{s}}\right)$ are, respectively, the transient responses of the process and the model to a unity step input, the identified model yields err $=1.15 \times 10^{-3}$, indicating good identification robustness. Then, assume that the noise level rises to NSR $=30 \%$ in terms of the measurement noise of $N\left(0, \sigma_{N}^{2}=0.572\right)$, the proposed IV identification method based on the above time interval of output data gives the process model, $G_{\mathrm{m}}=1.0541 \mathrm{e}^{-9.2686 s} / \mathrm{s}(22.2082 s+1)$, corresponding to err $=2.82 \times 10^{-2}$.

Example 2: Consider the SOPDT stable process studied in [27]

$$
G=\frac{\mathrm{e}^{-s}}{(s+1)^{2}} .
$$

Based on a biased relay test with $u_{+}=1.3, u_{-}=-0.7$, and $\varepsilon_{+}=-\varepsilon_{-}=0.2$, the proposed identification method composed of (19), (22), and (23) gives the process model listed in Table I, together with the intermediate values of the limit cycle for computation. It is seen that the proposed algorithm results in good accuracy.

To demonstrate identification robustness in the presence of measurement noise, random noise of $N\left(0, \sigma_{N}^{2}=0.00675\right)$, causing NSR $=20 \%$, as used by Panda and Yu [27], is added to the process-output measurement, which is then used for the relay feedback. Based on the averaging for five oscillation periods in the time interval of $[10,50] \mathrm{s}$, the limit-cycle data are computed as $\bar{P}_{\mathrm{u}}=5.33, \bar{A}_{\mathrm{u}}=0.4207$, and $\bar{\varphi}_{\mathrm{u}}=-2.9194$. Accordingly, the proposed algorithm yields the process model, $G_{\mathrm{m}}=1.0083 \mathrm{e}^{-1.0033 s} /(1.0028 s+1)^{2}$, indicating improved identification robustness as compared to that of Panda and $\mathrm{Yu}$ [27]. 
TABLE II

TEMPERATURE-RESPONSE MODELS FOR ZONES 1-3

\begin{tabular}{ccccccccc}
\hline & \multicolumn{2}{c}{ Heating-up Model } & & \multicolumn{2}{c}{ Relay Test } & \multicolumn{2}{c}{ Model for Steady Operation } \\
\cline { 2 - 7 } Zone & Proposed method & Z-N method & $P_{\mathrm{u}}$ & $A_{\mathrm{u}}$ & $\varphi_{\mathrm{u}}$ & Integrating Type & Stable Type \\
\hline \multirow{2}{*}{1} & $\frac{0.2842 e^{-33.23 s}}{s(147.1585 s+1)}$ & $\frac{0.1337 e^{-75 s}}{s}$ & 367.4 & 7.9775 & -2.9144 & $\frac{0.2046 e^{-29.4 s}}{s(65.3475 s+1)}$ & $\frac{62.6212 e^{-29.4 s}}{(153.0371 s+1)^{2}}$ \\
2 & $\frac{0.1719 e^{-25.01 s}}{s(56.7819 s+1)}$ & $\frac{0.1463 e^{-63 s}}{s}$ & 374.4 & 9.0318 & -2.8298 & $\frac{0.2159 e^{-27.8 s}}{s(60.4314 s+1)}$ & $\frac{62.7337 e^{-27.8 s}}{(145.2998 s+1)^{2}}$ \\
3 & $\frac{0.197 e^{-25.04 s}}{s(78.52 s+1)}$ & $\frac{0.1431 e^{-68 s}}{s}$ & 302.4 & 4.8005 & -2.8246 & $\frac{0.1386 e^{-23.4 s}}{s(46.4496 s+1)}$ & $\frac{31.4213 e^{-23.4 s}}{(113.3369 s+1)^{2}}$ \\
\hline
\end{tabular}

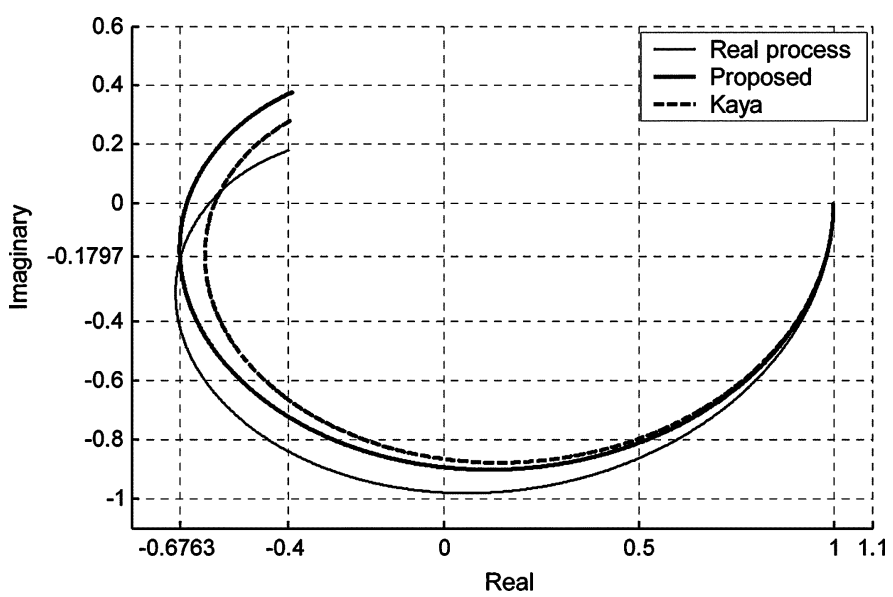

Fig. 5. Nyquist plots of model fitting for Example 4.

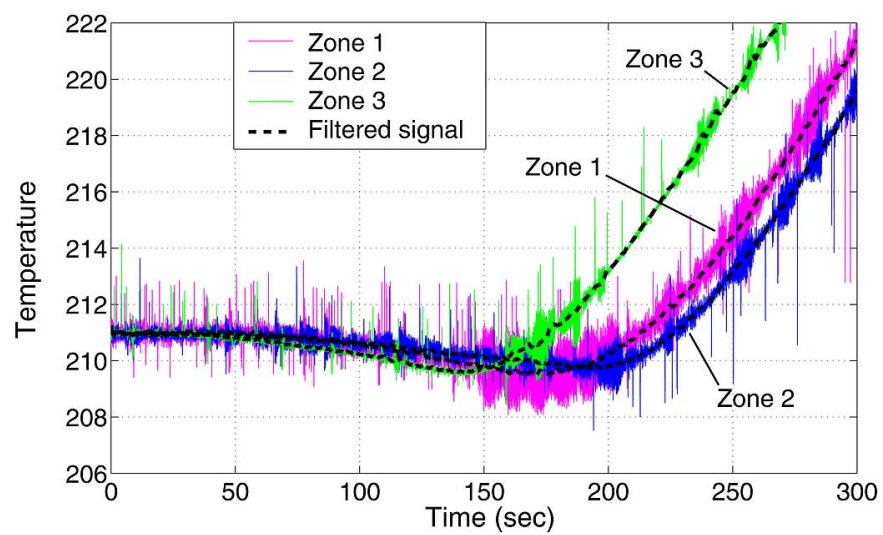

Fig. 6. Step-response tests for zones $1-3$.

Example 3: Consider the high-order integrating process studied in [28]

$$
G=\frac{(-s+1) \mathrm{e}^{-5 s}}{s(s+1)^{5}}
$$

Based on the process step-response data, Ingimundarson and Hägglund [28] gave an FOPDT model, $G_{\mathrm{m}}=$ $1.0000 \mathrm{e}^{-11.0000 s} / \mathrm{s}$. Using the measured data in the time interval of $[10,30] \mathrm{s}$ of the unity step response, the proposed identification method composed of (4), (5), (6), and (7) gives a SOPDT model, $G_{\mathrm{m}}=0.9936 \mathrm{e}^{-9.4145 s} / s(1.7364 s+1)$. For comparison, by letting $\tau_{\mathrm{p}}=0$, the proposed algorithm can also be used to obtain an FOPDT model, $G_{\mathrm{m}}=0.9811 \mathrm{e}^{-10.9029 s} / \mathrm{s}$. The Nyquist plots of these models are shown in Fig. 4. It can be seen that the proposed FOPDT model procures improved fitting as compared to that of Ingimundarson and Hägglund [28], and the proposed SOPDT model obtains apparently better fitting.

Example 4: Consider the third-order process studied in [29]

$$
G=\frac{\mathrm{e}^{-0.5 s}}{(s+1)\left(s^{2}+s+1\right)} \text {. }
$$

Based on a biased relay-feedback test, Kaya and Atherton [29] derived a critically damped SOPDT model, $G_{\mathrm{m}}=$ $1.00 \mathrm{e}^{-1.633 s} /(0.785 s+1)^{2}$. For comparison, using a biased relay test as in Example 2, the proposed algorithm composed of (19), (22), and (23) gives the process model listed in Table I. The Nyquist plots of the two models are shown in Fig. 5, which indicates again that improved fitting is captured by the proposed SOPDT model, particularly for the low-frequency range primarily referred to control-system design [18], [19]. Note that the proposed model response at $(-0.6763,-j 0.1797)$ corresponding to the oscillation frequency coincides precisely with the real process.

\section{EXPERIMENTAL Tests}

Consider the barrel-temperature control of an industrial Chen-Hsong reciprocating-screw injection-molding machine (model no. JM88-MKIII-C), of which the schematic diagram is shown in Fig. 1. There are six temperature zones in the heating barrel. Each zone is equipped with an electric heater with a capacity of $1040 \mathrm{~W}$, which is regulated via a zero-crossing solid state relay with pulse width modulation. Each zone temperature is measured using a K-type thermocouple. A 16-bit data acquisition card (AT-MIO-16X) of National Instruments is used for analog-to-digital and digital-to-analog conversions. The semicrystalline material of high-density polyethylene is used for the injection-molding experiment. According to the process characteristics, the rear three zones, 4-6, are primarily for heating the raw materials below a temperature of $200^{\circ} \mathrm{C}$, while the front three zones, $1-3$, are required to reach the melting temperature of $220{ }^{\circ} \mathrm{C}$ and tightly maintain the temperature, 


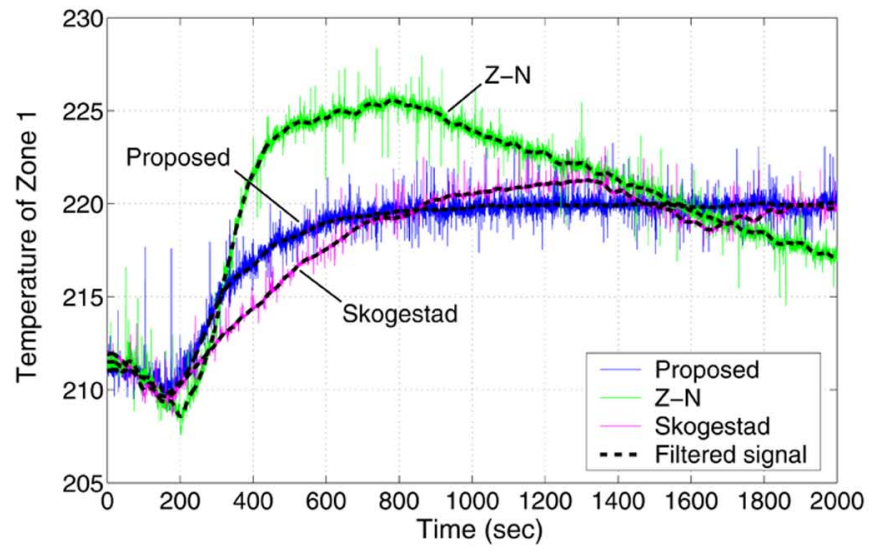

(a)

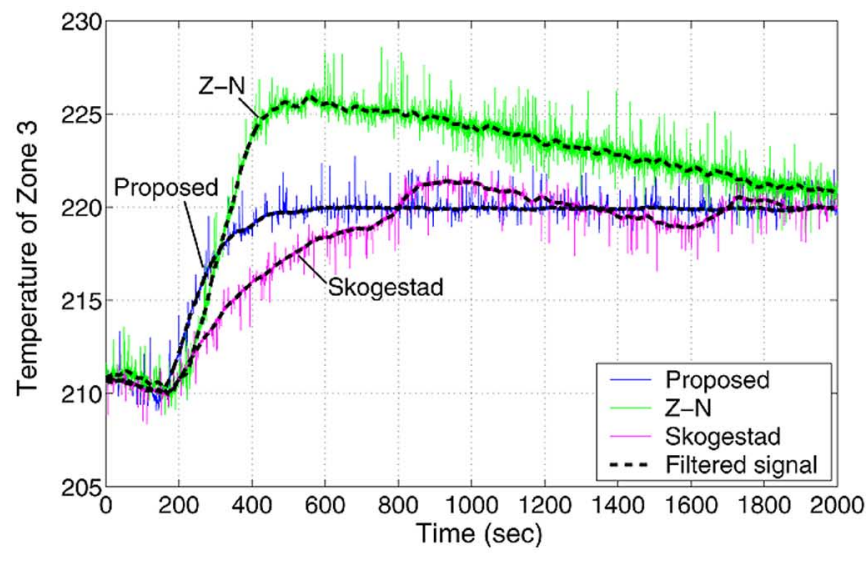

(c)

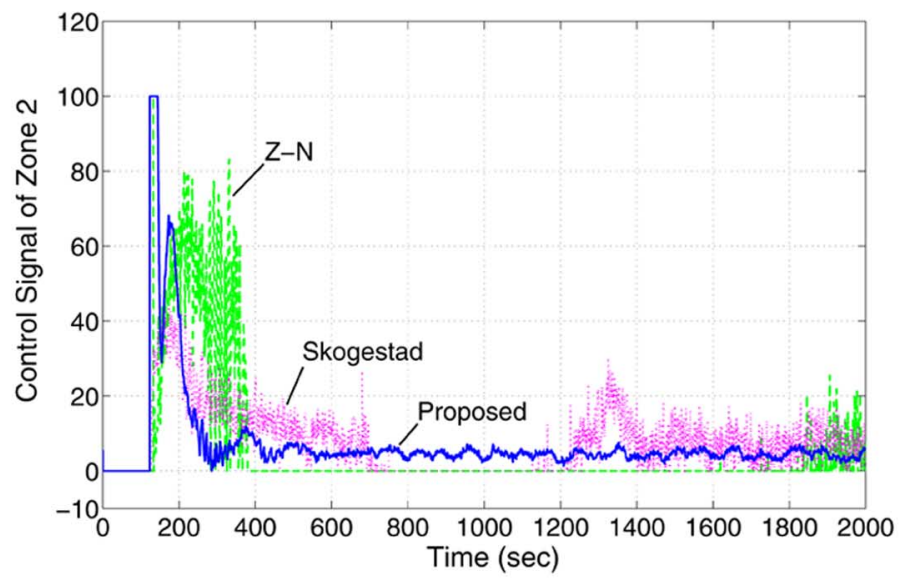

(e)

Fig. 7. (a)-(c) Heating-up responses and (d)-(f) control signals of zones 1-3.

e.g., within $\pm 0.5^{\circ} \mathrm{C}$, for injection molding. Therefore, identification and autotuning are herein focused on the front zones 1-3 for illustration. It should be noted that all the temperature zones have positive correlations with each other for the heating-up process and the injection-molding process of cycling, individual loop-control structure (i.e., multiloop control) is adopted for simplicity. The following identification procedure also takes

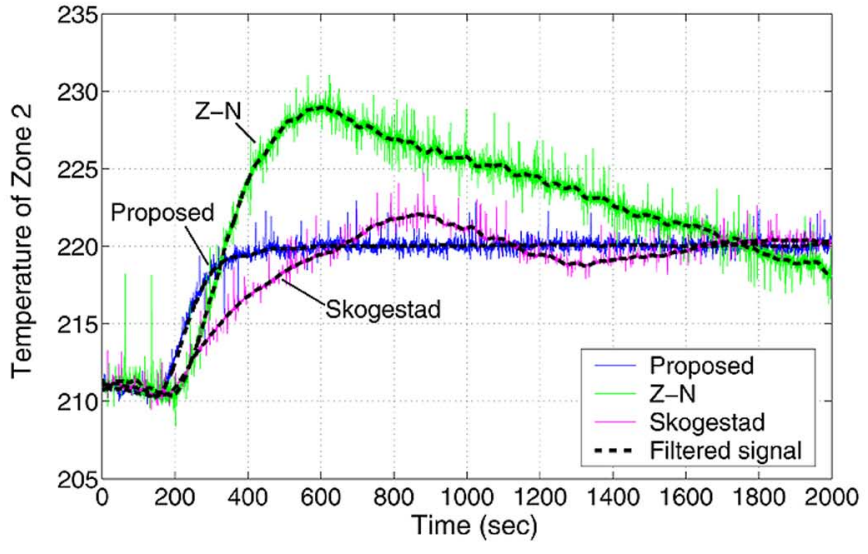

(b)

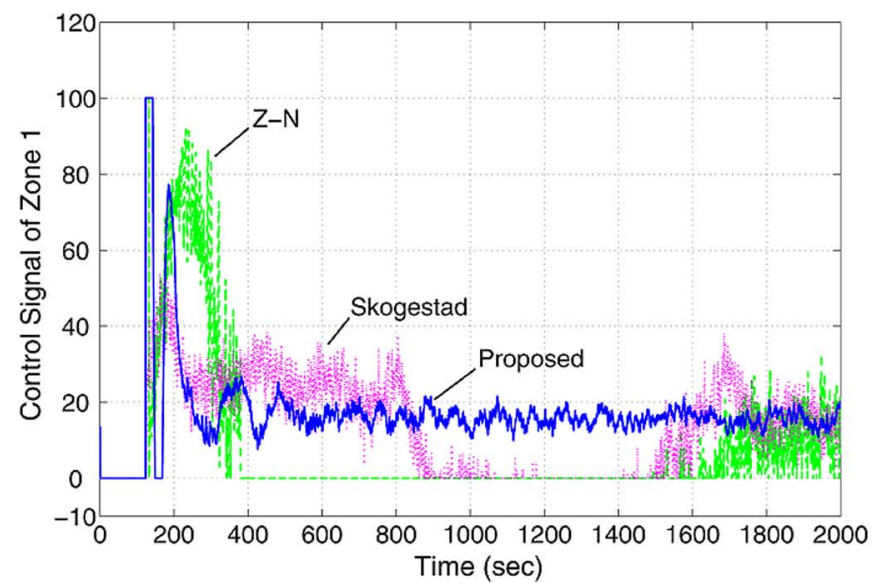

(d)

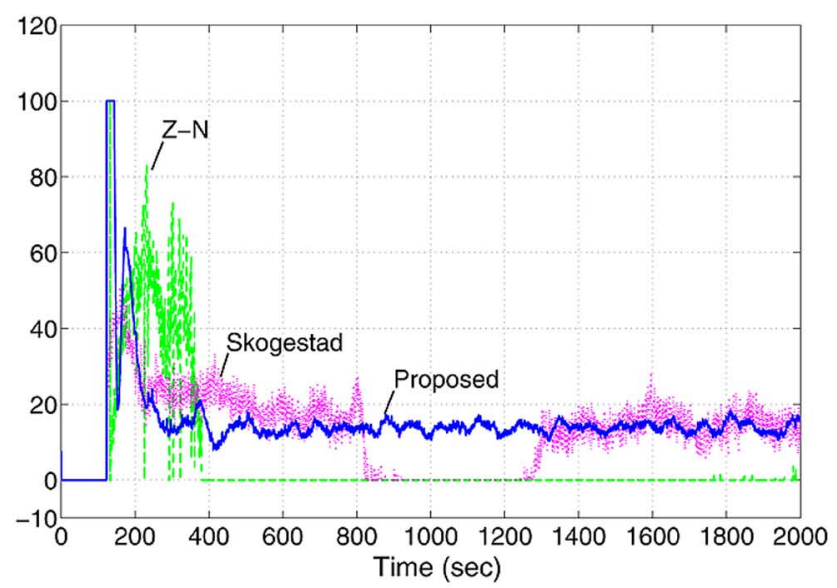

(f)

into account the positive correlation between the temperature zones.

To identify an integrating SOPDT model for representing the heating-up response characteristics for each of the three zones, an open-loop step-response test is conducted for each zone: First, the three zones are heated to a temperature over $210^{\circ} \mathrm{C}$, and then, the corresponding heaters are shut off. When the tem- 

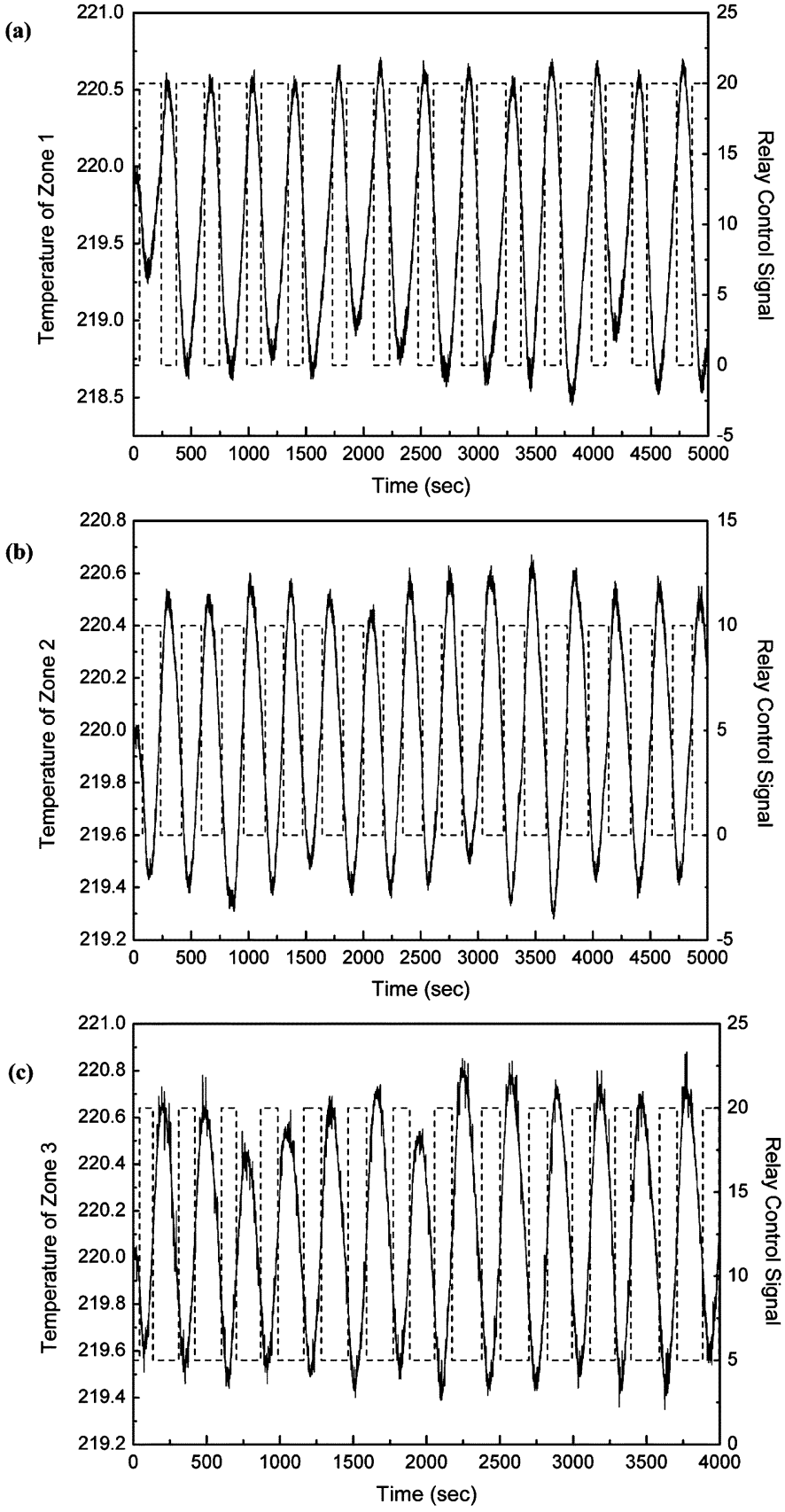

Fig. 8. Relay-identification tests for zones 1-3.

perature of the zone to be identified drops to $210{ }^{\circ} \mathrm{C}$, the three zones are simultaneously heated with the heaters turned fully on until the temperature of the zone to be identified reaches the set-point temperature of $220^{\circ} \mathrm{C}$. The experimental results with a sampling period of $200 \mathrm{~ms}$ are shown in Fig. 6. It can be seen that, due to air convection, the temperatures of zones 1-3 drop slowly before increasing to $220^{\circ} \mathrm{C}$. The time of the temperature drop can be viewed as a time delay of the heating-up response, which may be referenced to choose the initial transient response point for model identification. To reduce the influence of the measurement noise, a third-order Butterworth filter with a cutoff frequency of $f_{\mathrm{c}}=0.5 \mathrm{~Hz}$ is used in both the forward and reverse directions to recover the noisy data for identification, (a)

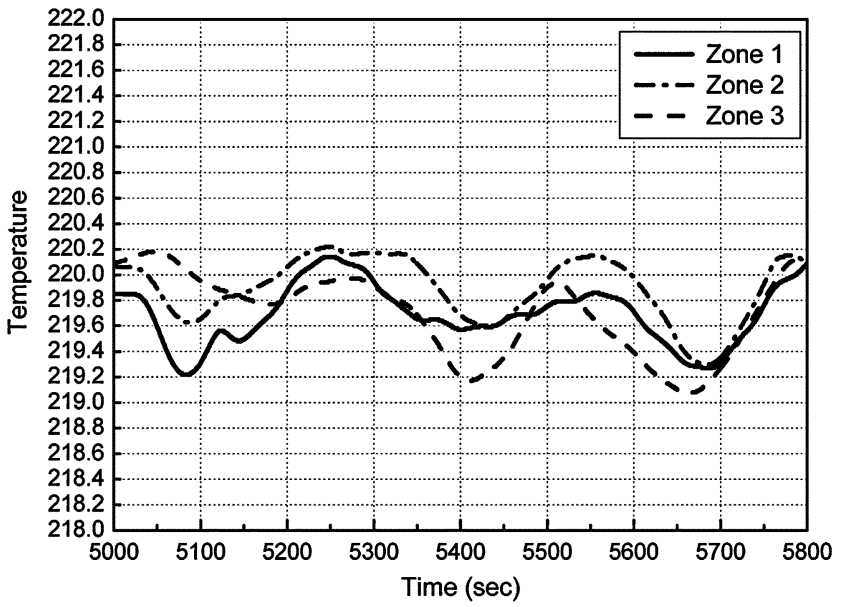

(b)

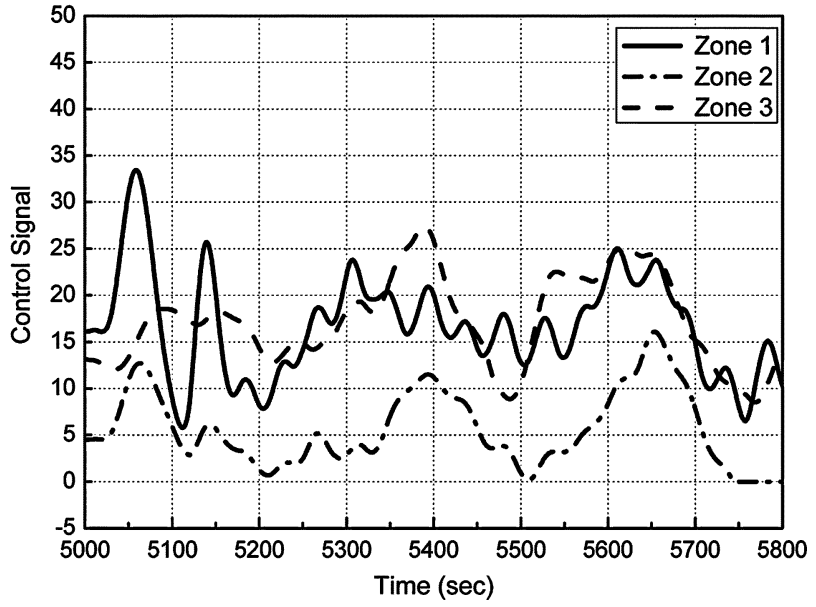

Fig. 9. Temperature responses of zones 1-3 using the heating-up models for injection molding.

shown as thick dashed lines in Fig. 6. By using the proposed IV identification method, the integrating SOPDT models of the three zones are obtained as listed in Table II, together with the integrating FOPDT models obtained in terms of the well-known Ziegler-Nichols (Z-N) step-response method [30].

Accordingly, the proposed control scheme with the controller-tuning formula of (31) is performed for a heating-up test from the temperature of $210{ }^{\circ} \mathrm{C}$. That is, zones $1-3$ are heated to slightly over $210{ }^{\circ} \mathrm{C}$, and then, the proposed control scheme is switched over after the temperature of any one of the three zones drops to $210^{\circ} \mathrm{C}$ via shutting off the corresponding heaters. The sampling period for control implementation is taken as $T_{\mathrm{s}}=0.5 \mathrm{~s}$, in view of that the maximal temperature increment $(\Delta T)$ is actually no greater than 0.1 ${ }^{\circ} \mathrm{C} / \mathrm{s}$, as can be verified from the filtered signals shown in Fig. 6. Correspondingly, the backward-discretization operator, $\dot{e}\left(k T_{\mathrm{s}}\right)=\left[e\left(k T_{\mathrm{s}}\right)-e\left((k-1) T_{\mathrm{s}}\right)\right] / T_{\mathrm{s}}$, is used for differential computation. To reduce the influence of measurement noise in the computation of the control output, an online noise-spike filtering strategy [31] is used for feedback control, i.e., the expression for $\hat{y}\left(k T_{\mathrm{s}}\right)$

$$
\begin{aligned}
& \hat{y}\left(k T_{\mathrm{s}}\right) \\
& = \begin{cases}y\left((k-1) T_{\mathrm{s}}\right)+\Delta T, & y\left(k T_{\mathrm{s}}\right)-y\left((k-1) T_{\mathrm{s}}\right) \geq \Delta T \\
y\left((k-1) T_{\mathrm{s}}\right)-\Delta T, & y\left(k T_{\mathrm{s}}\right)-y\left((k-1) T_{\mathrm{s}}\right) \leq-\Delta T \\
y\left(k T_{\mathrm{s}}\right), & \text { otherwise }\end{cases}
\end{aligned}
$$


(a)

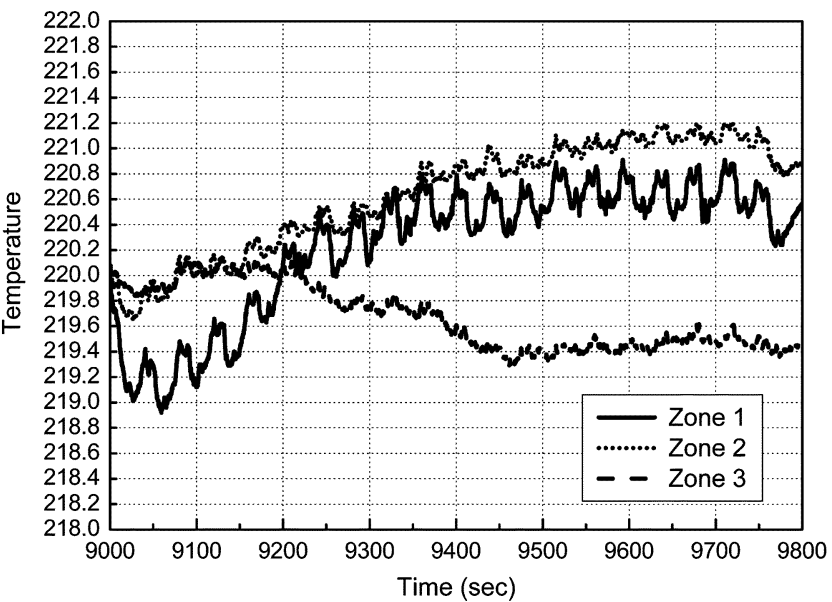

(b)

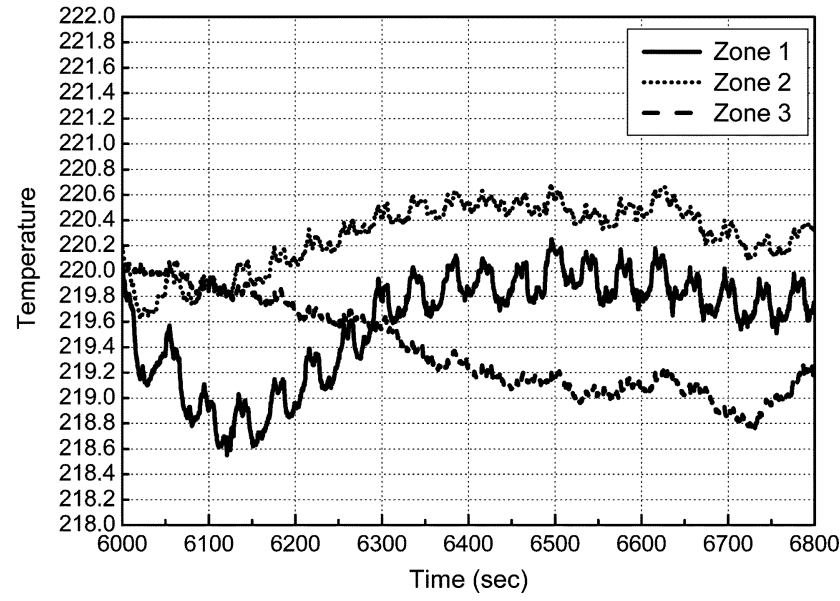

Fig. 10. Temperature responses of zones 1-3 in terms of (a) Z-N and (b) Skogestad methods.

where $\hat{y}\left(k T_{\mathrm{S}}\right)$ is the filtered temperature for feedback control, $y\left(k T_{\mathrm{S}}\right)$ is the measured temperature, and $\Delta T=0.005^{\circ} \mathrm{C}$ is the threshold for filtering noise. By setting the control parameters to $\lambda_{\mathrm{h}-1}=\lambda_{\mathrm{h}-2}=\lambda_{\mathrm{h}-3}=20$, respectively, for zones $1-3$, the experimental results are obtained as shown in Fig. 7. It can be seen that quick heating-up without temperature overshoot has been obtained for the three zones, compared to the well-known Z-N PID tuning method [30] based on the integrating FOPDT models listed in Table II. For further comparison, using the proposed integrating SOPDT model, the control results in terms of the recent IMC-based PID tuning method [32] with the parameter settings of $\tau_{\mathrm{c}-1}=\tau_{\mathrm{c}-2}=\tau_{\mathrm{c}-3}=30$ are also shown in Fig. 7. Note that the control signals have an implementational range of $[0,100]$, corresponding to heating power in the range of $0 \%-100 \%$. This implies that no negative control output can be used to drop the temperature. In the case of overheating, only load disturbance from air convection can help to drop the temperature, unavoidably leading to a prolonged settling time. From the tuning formula given in Section III.A, the heating-up time for each of zones 1-3 can be quantitatively evaluated, as can be verified from Fig. 7(a)-(c). Besides, it is shown in Fig. 7(d)-(f) that after heating-up, each of the control outputs maintains a constant value to balance the heat loss from air convection.

To identify the temperature-response models of zones $1-3$ for steady-state operation, online relay tests are conducted for the three zones, respectively. The results are shown in Fig. 8. Note (a)

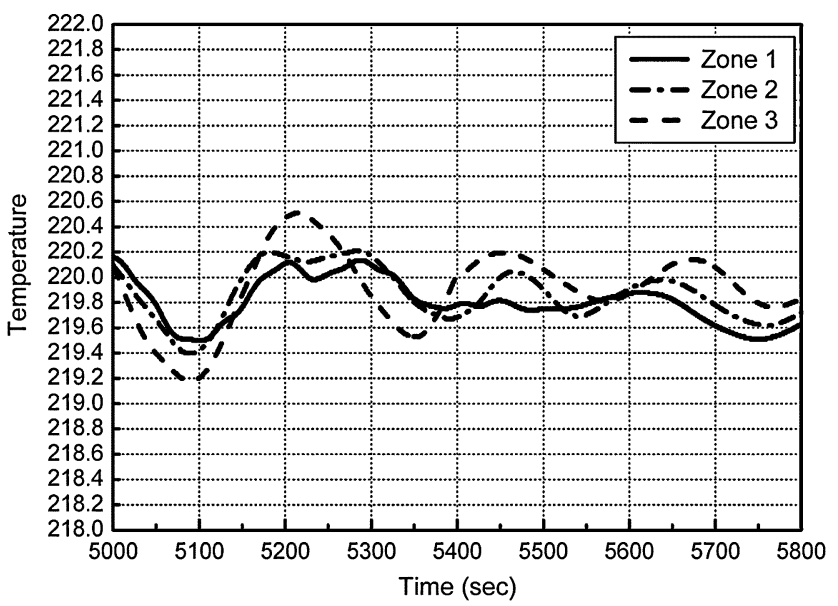

(b)

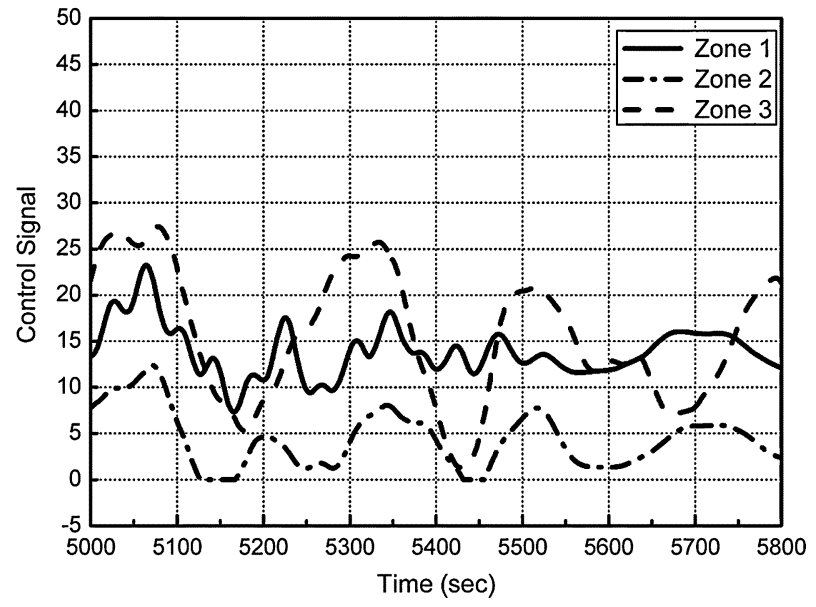

Fig. 11. Temperature responses of zones 1-3 using the stable models for injection molding.

that $u_{+}=20$ means $20 \%$ of the heating power and $u_{-}=0$ indicates shutting off the heater. The temperatures of $220.1^{\circ} \mathrm{C}$ and $219.8^{\circ} \mathrm{C}$ correspond to the relay-switch conditions of $\varepsilon_{+}=$ 0.1 and $\varepsilon_{-}=-0.2$, respectively. A third-order Butterworth filter with a cutoff frequency of $f_{\mathrm{c}}=0.5 \mathrm{~Hz}$ is used for the relayfeedback control against measurement noise, in view of that it can result in more regular oscillation periods as compared to the earlier noise-spike filter. To reduce the interaction between the three zones during a relay test for each zone, the other two zones are similarly heated or cooled following the relay-switch conditions for the zone to be identified. By averaging five similar oscillation periods for frequency-response estimation and using the measured time delay in the initial response of each relay test, the proposed relay-identification method gives the models of stable and integrating types listed in Table II, together with the limit-cycle data.

Based on the identified stable and integrating models, the proposed control scheme with the controller-tuning formula of (39) (or (31) for the integrating models) is performed for injection molding at the set-point temperature of $220^{\circ} \mathrm{C}$. The mold shape is rectangular with a length of $200 \mathrm{~mm}$, width of $150 \mathrm{~mm}$, and thickness of $2 \mathrm{~mm}$. The cycle time for yielding a product with a weight about $27.8 \mathrm{~g}$ is $40 \mathrm{~s}$. For illustration, 20 cycles are run to test the achievable control performance. First, the experimental results in terms of the heating-up model and the other two tuning methods of Z-N [30] and Skogestad [32] are shown, 
(a)

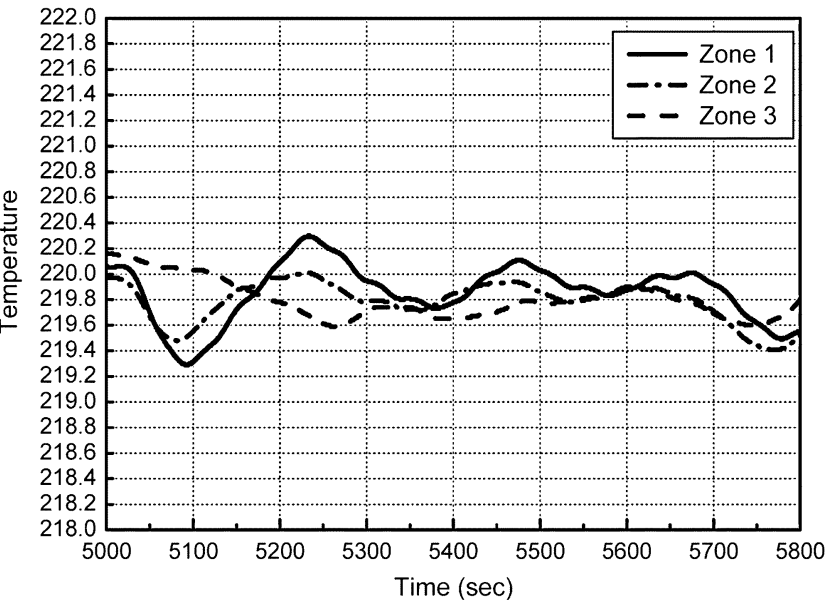

(b)

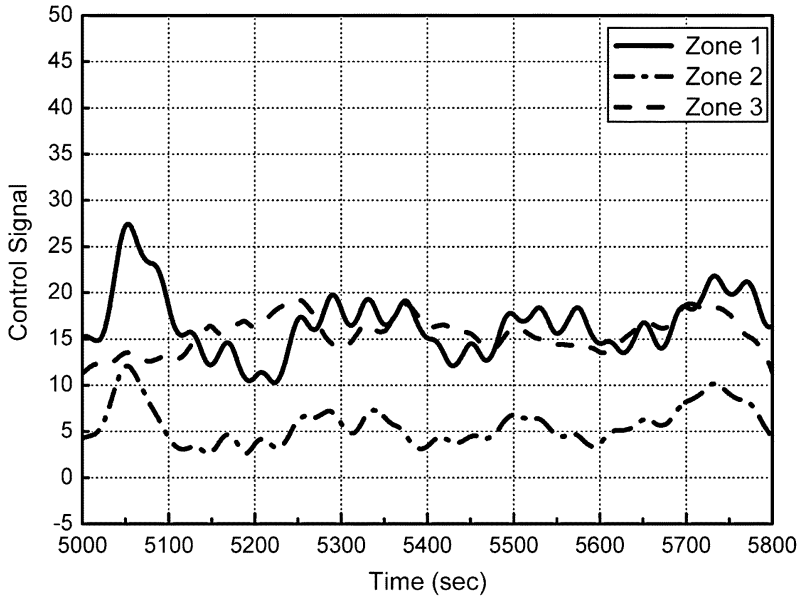

Fig. 12. Temperature responses of zones $1-3$ using the integrating models for injection molding.

respectively, in Figs. 9 and 10. Note that the starting time for each test is taken in terms of that all the three zone temperatures have been simultaneously maintained around $220^{\circ} \mathrm{C}$, and only filtered temperature responses are shown for clarity. Then, with the same tuning parameters, the experimental results in terms of the identified stable and integrating models from the relay test are shown, respectively, in Figs. 11 and 12. It can be seen that with both types of the zone models obtained from the relay tests, the zone temperatures are well maintained within the error band of $\pm 0.5^{\circ} \mathrm{C}$, except for the initial load-disturbance response for injection molding. Besides, it is seen that the temperature responses in terms of the heating-up integrating models are somewhat oscillatory as compared to using the stable and integrating models from the relay tests, due to less accuracy of representing the process-response characteristics around the set-point temperature of $220^{\circ} \mathrm{C}$, but are still acceptable from a practical view.

\section{CONCLUSION}

For temperature-control design, an identification method based on the step response has been proposed for obtaining the heating-up model, and another identification method based on a relay test around the set-point temperature has been given for obtaining the model of stable or integrating type for load-disturbance rejection. Both identification methods are capable of good robustness against measurement noise. A united control scheme based on the IMC structure has been proposed for both heating-up and steady-state load-disturbance rejection, which is capable of no overheating (i.e., temperature overshoot) for heating-up. Analytical controller-design formulas and quantitative tuning guidelines have been given for implementation of the desirable heating-up response and load-disturbance response, together with robust tuning constraints to accommodate for process uncertainties. The application to the barrel-temperature control of an injection-molding machine has well demonstrated the effectiveness of the proposed identification methods and control scheme.

\section{REFERENCES}

[1] M. Huzmezana, W. A. Goughb, G. A. Dumonta, and S. Kovac, "Time delay integrating systems: A challenge for process control industries. A practical solution," Control Eng. Pract., vol. 10, no. 10, pp. 1153-1161, Oct. 2002.

[2] K. Yao and F. Gao, "Optimal start-up control of injection molding barrel temperature," Polym. Eng. Sci., vol. 47, no. 3, pp. 254-261, Mar. 2007.

[3] C. H. Lu and C. C. Tsai, "Adaptive decoupling predictive temperature control for an extrusion barrel in a plastic injection molding process," IEEE Trans. Ind. Electron., vol. 48, no. 5, pp. 968-975, Oct. 2001.

[4] T. L. Chia, "Model predictive control helps to regulate slow processes-robust barrel temperature control," ISA Trans., vol. 41, no. 4, pp. 501-509, Oct. 2002.

[5] S. N. Huang, K. K. Tan, and T. H. Lee, "Adaptive GPC control of melt temperature in injection moulding," ISA Trans., vol. 38, no. 4, pp. 361-373, Nov. 1999.

[6] U. C. Moon, "A practical multiloop controller design for temperature control of a TV glass furnace," IEEE Trans. Control Syst. Technol., vol. 15, no. 6, pp. 1137-1142, Nov. 2007.

[7] D. B. Kaymak and W. L. Luyben, "Comparison of two types of twotemperature control structures for reactive distillation columns," Ind. Eng. Chem. Res., vol. 44, no. 13, pp. 4625-4640, 2005.

[8] E. A. Wolff and S. Skogestad, "Temperature cascade control of distillation columns," Ind. Eng. Chem. Res., vol. 35, no. 2, pp. 475-484, 1996.

[9] K. S. Lee, J. Lee, I. Chin, J. Choi, and J. H. Lee, "Control of wafer temperature uniformity in rapid thermal processing using an optimal iterative learning control technique," Ind. Eng. Chem. Res., vol. 40, no. 7, pp. 1661-1672, 2001.

[10] P. Valigi, M. L. Fravolini, and A. Ficola, "Improved temperature control of a batch reactor with actuation constraints," Control Eng. Pract., vol. 14, no. 7, pp. 783-797, Jul. 2006.

[11] M. Shahrokhi and A. Nejati, "Optimal temperature control of a propane thermal cracking reactor," Ind. Eng. Chem. Res., vol. 41, no. 25, pp. 6572-6578, 2002.

[12] E. Grassi and K. Tsakalis, "PID controller tuning by frequency loopshaping: Application to diffusion furnace temperature control," IEEE Trans. Control Syst. Technol., vol. 8, no. 5, pp. 842-847, Sep. 2000.

[13] C. Diduch, R. Dubay, and W. G. Li, "Temperature control of injection molding. Part I: Modeling and identification," Polym. Eng. Sci., vol. 44, no. 12, pp. 2308-2317, Dec. 2004.

[14] R. Dubay, C. Diduch, and W. G. Li, "Temperature control of injection molding. Part II: Controller design, simulation, and implementation," Polym. Eng. Sci., vol. 44, no. 12, pp. 2318-2326, Dec. 2004

[15] E. Dassau, B. Grosman, and D. R. Lewin, "Modeling and temperature control of rapid thermal processing," Comput. Chem. Eng., vol. 30, no. 4, pp. 686-697, Feb. 2006.

[16] D. Sankowski, J. Kucharski, and W. Lobodzinski, "Autotuning temperature control using identification by multifrequency binary sequences," Proc. Inst. Elect. Eng.-Control Theory Appl., vol. 144, no. 3, pp. 233-240, May 1997.

[17] K. K. Tan, Q. G. Wang, T. H. Lee, and C. H. Gan, "Automatic tuning of gain-scheduled control for asymmetrical processes," Control Eng. Pract., vol. 6, no. 11, pp. 1353-1363, Nov. 1998.

[18] Q. G. Wang, T. H. Lee, and C. Lin, Relay Feedback: Analysis, Identification and Control. London, U.K.: Springer-Verlag, 2003.

[19] C. C. Yu, Autotuning of PID Controllers: A Relay Feedback Approach, 2nd ed. London, U.K.: Springer-Verlag, 2006.

[20] T. Liu and F. Gao, "Identification of integrating and unstable processes from relay feedback," Comput. Chem. Eng., vol. 32, no. 12, pp. 3038-3056, Dec. 2008 
[21] T. Soderstrom and P. Stoica, System Identification. New York: Prentice-Hall, 1989.

[22] L. Ljung, System Identification: Theory for the User, 2nd ed. Englewood Cliff, NJ: Prentice-Hall, 1999.

[23] T. Liu, Y. Z. Cai, D. Y. Gu, and W. D. Zhang, "New modified Smith predictor scheme for controlling integrating and unstable processes," Proc. Inst. Elect. Eng.-Control Theory Appl., vol. 152, no. 2, pp. 238-246, Mar. 2005.

[24] M. Morari and E. Zafiriou, Robust Process Control. Englewood Cliffs, NJ: Prentice-Hall, 1989.

[25] K. M. Zhou, J. C. Doyle, and K. Glover, Robust and Optimal Control. Englewood Cliffs, NJ: Prentice-Hall, 1995.

[26] I. Kaya, "Parameter estimation for integrating processes using relay feedback control under static load disturbances," Ind. Eng. Chem. Res., vol. 45, no. 13, pp. 4726-4731, 2006.

[27] R. C. Panda and C. C. Yu, "Shape factor of relay response curves and its use in autotuning," J. Process Control, vol. 15, no. 8, pp. 893-906, Dec. 2005.

[28] A. Ingimundarson and T. Hägglund, "Robust tuning procedures of dead-time compensating controllers," Control Eng. Pract., vol. 9, no. 11, pp. 1195-1208, Nov. 2001.

[29] I. Kaya and D. P. Atherton, "Parameter estimation from relay autotuning with asymmetric limit cycle data," J. Process Control, vol. 11, no. 4, pp. 429-439, Aug. 2001.

[30] K. J. Asström and T. Hägglund, PID Controllers, 2nd ed. Research Triangle Park, NC: ISA Soc. Amer., 1995.

[31] D. E. Seborg, T. F. Edgar, and D. A. Mellichamp, Process Dynamic and Control, 2nd ed. Hoboken, NJ: Wiley, 2004.

[32] S. Skogestad, "Simple analytical rules for model reduction and PID controller tuning," J. Process Control, vol. 13, no. 4, pp. 291-309, Jun. 2003.

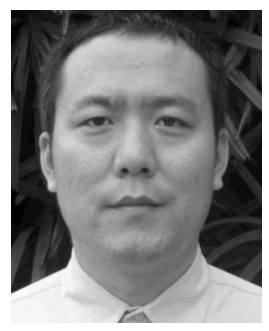

Tao Liu was born in Kuerle, Xinjiang, China, in 1974. He received the B.Eng. and M.S. degrees in electrical engineering from Chongqing University, Chongqing, China, in 1996 and 2002, respectively, and the Ph.D. degree in control science and engineering from Shanghai Jiaotong University, Shanghai, China, in 2006.

Since 2006, he has been a Postdoctoral Research Fellow with the Department of Chemical and Biomolecular Engineering, Hong Kong University of Science and Technology, Kowloon, Hongkong. His research interests include process identification/modeling, control-system design, autotuning, batch process optimization, and robust control theory.

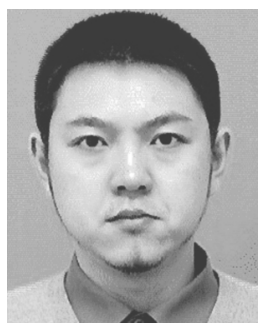

Ke Yao received the B.Eng. and M.S. degrees in control theory and engineering from Northeastern University, Shenyang, China, in 1999 and 2002, respectively, and the Ph.D. degree in chemical engineering from Hong Kong University of Science and Technology (HKUST), Kowloon, Hong Kong, in 2008

Since 2008, he has been a Research Associate with the Center for Polymer Processing and Systems, HKUST. His research interests include process control, system optimization, and injection-molding technology.

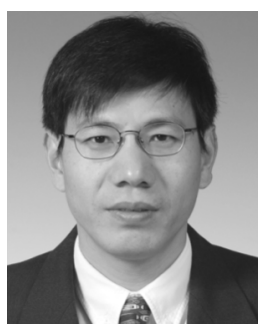

Furong Gao received the B.Eng. degree in automation from China University of Petroleum, Dongying, China, in 1985 and the M.Eng. and Ph.D. degrees in chemical engineering from McGill University, Montreal, QC, Canada, in 1989 and 1993, respectively.

He was a Senior Research Engineer with Moldflow International Company Ltd. Since 1995, he has been with Hong Kong University of Science and Technology, Kowloon, Hong Kong, where he is currently a Professor with the Department of Chemical and Biomolecular Engineering. His research interests are in the areas of process monitoring and control and polymer processing. 